\title{
COLETA DE SÊMEN OVINO EM ESTAÇÃO OU DECÚBITO LATERAL UTILIZANDO DIFERENTES ELETROEJACULADORES
}

\author{
Ligia Freitas de Lima, ${ }^{1}$ Rodrigo Dorneles Tortorella, ${ }^{2}$ Diogo Ramos Leal, ${ }^{2}$ \\ Pedro Ivo Braga Passos, ${ }^{2}$ Roberto Sartori, ${ }^{4}$ Thiago Antônio de Souza Nascimento Silva, ${ }^{5}$ \\ Hetielle Harumi Hashimoto ${ }^{6}$ Jairo Pereira Neves, ${ }^{3}$ \\ 1. Mestranda em Ciências Agrárias - UnB \\ 2. Graduandos FAV/UnB \\ 3. Professor doutor, FAV/UnB. E-mail: jpneves@unb.br \\ 4. Pesquisador EMBRAPA/ Recursos Genéticos \\ 5. Mestre, FAV/UnB \\ 6. Graduando FAV/UnB.
}

RESUMO

O experimento teve como objetivo avaliar e comparar dois eletroejaculadores (EEr): um automático (A - Biocom Ltda., Uberaba, MG) e outro manual (M - Duboi, Campo Grande, MS), utilizando dois métodos de contenção: estação (E) e decúbito lateral (D). Foram utilizados doze carneiros: Santa-Inês ( $n=9)$, Ile-de-France $(n=2)$ e Texel $(n=1)$. O sêmen foi coletado duas vezes por semana e as coletas realizadas três vezes em cada grupo ( $\mathrm{AD}$ - automático em decúbito; $\mathrm{AE}$ - automático em estação; $\mathrm{MD}$ - manual em decúbito e ME - manual em estação) resultando em doze coletas. Antes e depois das coletas de sêmen, amostras de sangue foram coletadas para análise dos níveis plasmáticos de cortisol com objetivo de verificação do estresse entre os grupos e indivíduos. Procedeu-se à avaliação das coletas pelo tempo em segundos para obtenção da fração seminal, tempo de duração da fração seminal e porcentagem de carneiros que expuseram o pênis. Os parâmetros avaliados referentes ao sêmen foram: volume, aspecto, motilidade espermática progressiva, turbilhonamento, vigor espermático, número total de espermatozoides no ejaculado e morfologia espermática. Em 7,1\% de todas as coletas não houve ejaculação, sendo que o grupo submetido ao tratamento $\mathrm{AD}(20 \%)$ foi responsável pela maior fração das coletas sem ejaculação $(\mathrm{P}<0,05)$. Os grupos não apresentaram diferenças significativas quanto ao aumento do nível de cortisol após as coletas $(\mathrm{P}>0,05)$. No entanto, houve diferença entre indivíduos $(\mathrm{P}<0,05)$. As coletas de sêmen nos animais em decúbito demandaram menor tempo para atingir a fração seminal e também proporcionaram maior duração da fração seminal $(\mathrm{P}<0,05)$. $\mathrm{O}$ grupo $\mathrm{MD}$ obteve menor tempo em atingir a fração seminal e produziu um maior volume de sêmen quando comparado ao grupo $\mathrm{AE}(\mathrm{P}<0,05)$. Os dados do experimento permitem concluir que o EEr automático e manual apresentaram resultados semelhantes, assim como as posições de coleta, decúbito ou estação.

PALAVRAS-CHAVES: Bem-estar animal, cortisol, eletroejaculação, estresse, sêmen.

\section{ABSTRACT}

\section{SEMEN COLLECTION IN RAMS STANDING UP OR IN LATERAL RECUMBENCY USING DIFFERENT ELECTROEJACULATORS}

The objective of the experiment was to evaluate and compare two electroejaculators: an automatic one (A - Biocom Ltda., Uberaba, MG) and a manual one (M - Duboi, Campo Grande, MS), using two methods of physical restrain: standing (E) or lateral recumbency (D). Twelve rams were used: Santa Inês ( $n=9)$,
Ile-de-France $(n=2)$ and Texel $(n=1)$. Semen were collected twice a week and each animal had the semen collected three times in each group ( $\mathrm{AD}$ - automatic in recumbency, $\mathrm{AE}$ - automatic in station, MD - manual in recumbency, and ME - manual in station) resulting in 12 collections. Blood samples were obtained before 
and after the semen collections to measure changes in circulating cortisol in order to measure stress among groups and individuals. The collections were evaluated per the time in seconds to obtain seminal fraction, time of seminal fraction and percentage of rams that exposed the penis. The collected semen parameters evaluated were: volume, aspect, progressive motility, wave motion, sperm vigor, total number of spermatozoa and sperm morphology. In $7.1 \%$ of the collections, there was no ejaculation, moreover, group AD was responsible for most of the collections without ejaculation (20\%) $(\mathrm{P}<0,05)$. There was no difference among groups $(\mathrm{P}>0.05)$ relating to cortisol level after collections. However, there was difference among individuals $(\mathrm{P}<0.05)$. The collections in lateral recumbency were faster regarding achievement of seminal fraction and provided more time length for seminal fraction. The MD group required a shorter time length to achieve the seminal fraction and produced a greater volume of ejaculate in comparison to the AS group. It can be concluded by these results that both eletroejaculators (automatic and manual) had similar results as well as both methods of physical restrain (standing and lateral recumbency).

KEYWORDS: Animal welfare, cortisol, electroejaculation, semen, stress.

\section{INTRODUÇÃO}

A eletroejaculação (EE) é um método eficaz para obtenção de amostras de sêmen para avaliação andrológica (PALMER, 2005), além de ser uma alternativa para animais que raramente têm contato com seres humanos. O uso da vagina artificial nestes casos poderá ser restrito, pela necessidade de um período de treinamento, ao qual muitas vezes os animais não se adaptam (ORTIZ DE MONTELLANO et al. 2006). A rejeição ao regime de coleta por vagina artificial é muito comum em animais de determinadas raças em extinção que necessitam do armazenamento de material genético em bancos de germoplasma.

A EE pode ocasionar algumas reações adversas como contrações musculares, agitação, vocalizações e ocasionalmente quedas. Estas possíveis situações são indicativas de desconforto, o que prejudica o bem-estar animal (PALMER, 2005).

Por ser dolorosa em seres humanos, MOSURE (1998) propôs que a EE sem anestesia poderia ser dolorosa também para os animais. Por esse motivo, PALMER (2005) a considera desumana, e, em consequência, a EE em animais não anestesiados foi banida em vários países europeus (MOSURE, 1998). Contudo, ORTIZ DE MONTELLANO et al. (2006) citaram testes de aversão realizados em bodes (CARTER et al., 1990), carneiros (COOK, 1996) e touros (BARTH \& BOWMAN, 1994), nos quais não foram demonstrados sinais de relutância aos tratadores e à contenção quando a EE era realizada com frequência.

Além das desvantagens citadas, tais como indução de dor e estresse no animal, o eletroejaculador (EEr) pode fornecer um ejaculado com características fora dos parâmetros fisiológicos da espécie (MIES
FILHO, 1987; REICHENBACH et al., 2008) e uma variação na resposta, dependendo da qualidade do equipamento e habilidade do operador. A utilização de um aparelho automático pré-programado para liberação de estímulos elétricos de forma crescente poderia reduzir o estresse do animal, proporcionar a obtenção de amostras mais homogêneas e um maior conforto ao operador.

Diante disso, este experimento teve o objetivo de avaliar e comparar um eletroejaculador automático (A - Biocom Ltda., Uberaba, MG) a outro manual (M - Duboi, Campo Grande, MS) associados a dois métodos de contenção, estação e decúbito lateral, para, dessa forma, subsidiar a escolha da melhor maneira de se aplicar a eletroejaculação, permitindo aumentar a qualidade do sêmen e minimizar o estresse sofrido pelos animais.

\section{MATERIAL E MÉTODOS}

Doze carneiros (9 Santa-Inês, 2 Ile-de-France, 1 Texel) tiveram o sêmen coletado duas vezes por semana, durante seis semanas, perfazendo um total de três coletas por animal em cada tratamento testado, conforme Figura 1.

Obtiveram-se, portanto, quatro tratamentos: $\mathrm{AE}$ - EEr automático em estação; $\mathrm{ME}$ - EEr manual em estação; AD - EEr automático em decúbito lateral; e MD - EEr manual em decúbito lateral (Figura 1). Todas as coletas foram cronometradas e não excederam quatro minutos.

O EEr automático é composto por um transdutor de $16 \mathrm{~cm}$ de comprimento; $5,5 \mathrm{~cm}$ de circunferência; $2,5 \mathrm{~cm}$ de diâmetro e três eletrodos longitudinais de $7,5 \mathrm{~cm}$. Já o EEr manual possui $20,0 \mathrm{~cm}$ 
de comprimento; $6,5 \mathrm{~cm}$ de circunferência; $3,0 \mathrm{~cm}$ de diâmetro e três eletrodos longitudinais de $7,0 \mathrm{~cm}$. Os eletrodos foram direcionados e mantidos voltados para a região ventral do animal durante toda a coleta.

Imediatamente antes e após quinze minutos de cada coleta de sêmen, foram obtidas amostras de sangue por venopunção da jugular em tubos heparinizados $\left(\right.$ BD Vacutainer ${ }^{\circledR}$ ) para a quantificação dos níveis plas- máticos de cortisol. Armazenaram-se as amostras sob refrigeração por um período não superior a duas horas até o momento da centrifugação para obtenção do plasma. A centrifugação foi realizada a $1500 \times \mathrm{g}$ durante quinze minutos e o plasma armazenado em duplicatas preservadas à temperatura de $-20^{\circ} \mathrm{C}$ para posterior análise com o uso do Coat-A-Count (Diagnostic Products Corporation, Los Angeles, CA, USA).

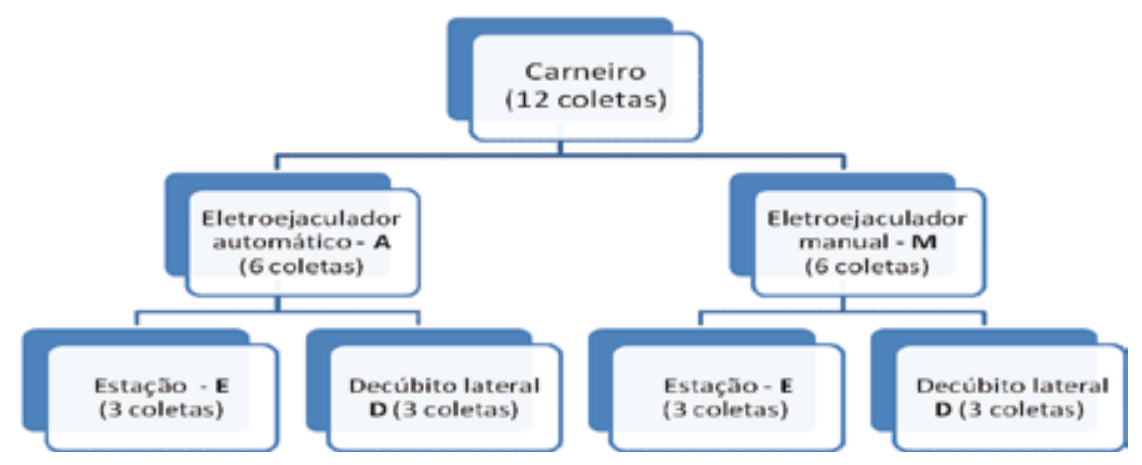

FIGURA 1. Tratamentos de coleta individual. Foram realizadas doze coletas por animal.

Durante cada coleta, procedeu-se a uma avaliação subjetiva do nível de estresse dos animais, atribuindo-se números de 0 a 3 ( 0 - ausente, 1 - baixo, 2 - médio, 3 - alto). Essa avaliação baseou-se nos graus de agitação, vocalizações e relutância observados durante o procedimento, conforme SARTORI et al. (2005). Para a coleta em estação, os animais foram contidos em um tronco com a ajuda de dois auxiliares. Nas coletas em decúbito lateral também foram necessários dois auxiliares para colocação dos animais em posição adequada e realizar a contenção dos membros pélvicos, torácicos e cabeça. O transdutor foi lubrificado com gel à base de carboximetilcelulose, e então inserido no reto de forma que os eletrodos ficassem voltados para a região ventral do animal.

Analisaram-se os seguintes parâmetros referentes à coleta: tempo em segundos para obtenção da fração seminal, tempo de duração da fração seminal e porcentagem de carneiros que expuseram o pênis. São os seguintes os itens avaliados referentes ao sêmen obtido: volume do sêmen ( $\mathrm{mL})$, aspecto do sêmen (1 - aquoso, 2 - leitoso, 3 - cremoso), motilidade espermática progressiva (\%), turbilhonamento (1 a 5), vigor espermático (1 a 5), número total de espermatozoides no ejaculado e morfologia espermática, conforme normas do CBRA (1998).

Compararam-se os parâmetros seminais e as concentrações séricas de cortisol por análise de variância (ANOVA), utilizando-se o proc GLM do programa estatístico SAS, e para comparação das médias, o teste de Tukey. $\mathrm{O}$ nível de significância adotado foi de $\mathrm{P}<0,05$.

\section{RESULTADOS E DISCUSSÃO}

Em virtude do óbito de um animal, não foi possível realizar três coletas, perfazendo no total 141 . Destas, em dez ocasiões $(7,1 \%)$ não se obteve ejaculado nos quatro minutos de estímulo elétrico, sendo que o grupo $\mathrm{AD}$ foi o que obteve o maior número de coletas frustradas, $20 \%(\mathrm{P}<0,05$, Tabela 1$)$.

A avaliação subjetiva do nível de estresse durante as coletas não apresentou diferença significativa entre os grupos ( $\mathrm{P}>0,05$, Tabela 1$)$. Contrariamente, SARTORI et al. (2005), em estudo para comparação do desempenho do mesmo EEr automático, na espécie 
bovina, diante de dois equipamentos manuais, constataram que o EEr automático gerou um nível mais baixo de estresse. Esse estudo utilizou os mesmos parâmetros de avaliação de estresse do presente trabalho.

A coleta de sêmen no grupo MD mostrou-se mais rápida $(\mathrm{P}<0,05$; Tabela 1$)$ em atingir a fração seminal e produziu um maior volume de ejaculado quando comparado ao grupo AE $(\mathrm{P}<0,01$; Tabela 2$)$. Porém esses resultados não produziram menor tempo de duração das coletas ou um maior número total de espermatozoides no ejaculado. Os demais grupos não diferiram entre si ( $\mathrm{P}>0,05$; Tabela 1$)$. SARTORI et al. (2005), em bovinos, não encontraram diferença no tempo para atingir a fração seminal entre o EEr automático e um EEr manual, porém o volume seminal, quando utilizado o automático, foi maior, assim como o número de espermatozoides no ejaculado desses animais. $\mathrm{O}$ menor tempo para atingir a fração seminal no grupo
MD pode ser devido a uma tendência de aceleração dos estímulos elétricos, já que o controle do EEr manual é dependente do treinamento do profissional.

Não foram observadas diferenças no aspecto do sêmen entre os grupos analisados $(\mathrm{P}>0,05$; Tabela 2). Outros parâmetros referentes ao sêmen obtido (turbilhonamento, motilidade espermática progressiva, vigor, total de anormalidades espermáticas, número total de espermatozoides no ejaculado) também não apresentaram diferenças entre os grupos $(\mathrm{P}>0,05$; Tabela 2).

Em relação ao aumento dos níveis plasmáticos de cortisol não se observaram diferenças entre os grupos ( $\mathrm{P}>0,05$; Tabela 3 ). Ou seja, tanto o EEr automático como o manual induziram níveis semelhantes de estresse aos animais, independentemente da posição em decúbito ou estação.

TABELA 1. Resultados (média \pm DP) dos parâmetros analisados referentes à coleta de sêmen nos carneiros $(\mathrm{n}=12$ por grupo) em estação (E) ou em decúbito (D) com o uso de eletroejaculador automático (A) ou manual (M)

\begin{tabular}{|c|c|c|c|c|}
\hline & \multicolumn{4}{|c|}{ Grupos } \\
\hline & $\mathrm{AE}$ & ME & $\mathrm{AD}$ & MD \\
\hline $\begin{array}{l}\text { Não obtenção de ejaculado após } 4 \text { minutos de estímulos } \\
(\%)\end{array}$ & $\begin{array}{c}5,9^{\mathrm{a}} \\
(2 / 35)\end{array}$ & $\begin{array}{c}0,0^{\mathrm{a}} \\
(0 / 35)\end{array}$ & $\begin{array}{l}20,0^{\mathrm{b}} \\
(7 / 35)\end{array}$ & $\begin{array}{c}5,6^{\mathrm{a}} \\
(2 / 36)\end{array}$ \\
\hline Nível de estresse ( 0 a 3 ) & $\begin{array}{l}1,2 \pm 0,5 \\
(\mathrm{n}=33)\end{array}$ & $\begin{array}{l}1,3 \pm 0,5 \\
(\mathrm{n}=35)\end{array}$ & $\begin{array}{l}1,2 \pm 0,4 \\
(\mathrm{n}=26)\end{array}$ & $\begin{array}{l}1,3 \pm 0,4 \\
(\mathrm{n}=31)\end{array}$ \\
\hline Tempo para atingir fração seminal (s) & $\begin{array}{c}76,7 \pm 46,9^{a} \\
(\mathrm{n}=32)\end{array}$ & $\begin{aligned} 64,4 & \pm 63,5^{\mathrm{ab}} \\
(\mathrm{n} & =35)\end{aligned}$ & $\begin{array}{l}64,4 \pm 40,5^{\mathrm{ab}} \\
\quad(\mathrm{n}=28)\end{array}$ & $\begin{array}{c}42,5 \pm 38,2^{b} \\
\quad(n=33)\end{array}$ \\
\hline Tempo para atingir fração pós-seminal (s) & $\begin{array}{l}146,6 \pm 47,1 \\
\quad(n=33)\end{array}$ & $\begin{array}{l}139,3 \pm 64,5 \\
(\mathrm{n}=35)\end{array}$ & $\begin{array}{l}127,9 \pm 46,3 \\
(\mathrm{n}=28)\end{array}$ & $\begin{array}{l}123,0 \pm 56,4 \\
(\mathrm{n}=33)\end{array}$ \\
\hline Exposição de pênis durante colheita (\%) & $\begin{array}{l}91,2 \pm 28,8 \\
\quad(n=34)\end{array}$ & $\begin{array}{c}91,2 \pm 28,8 \\
(\mathrm{n}=34)\end{array}$ & $\begin{array}{c}86,2 \pm 35,1 \\
(n=29)\end{array}$ & $\begin{array}{c}91,2 \pm 28,8 \\
(\mathrm{n}=34)\end{array}$ \\
\hline
\end{tabular}

$\mathrm{a}, \mathrm{b}=$ letras minúsculas diversas entre colunas determina diferença significativa entre tratamentos $(\mathrm{P} \leq 0,05)$.

TABELA 3. Concentração plasmática de cortisol (média \pm DP) nos carneiros $(n=12$ por grupo) colhidos nos quatro grupos

\begin{tabular}{lccccc}
\hline & & \multicolumn{3}{c}{ Grupos } \\
\cline { 3 - 6 } & & $\mathrm{AE}$ & $\mathrm{ED}$ & $\mathrm{AD}$ \\
$(\mathrm{n}=23)$ & $(\mathrm{n}=24)$ & $\begin{array}{c}\mathrm{DM} \\
(\mathrm{n}=24)\end{array}$ \\
\hline $\begin{array}{l}\text { Cortisol } \\
\text { Plasmático }\end{array}$ & Antes da colheita seminal & $13,7 \pm 15,9$ & $11,6 \pm 7,3$ & $8,7 \pm 6,3$ & $11,2 \pm 9,5$ \\
$(\mathrm{ng} / \mathrm{mL})$ & Após a colheita seminal & $49,7 \pm 17,4$ & $45,6 \pm 11,6$ & $45,7 \pm 21$ & $50,2 \pm 14,3$ \\
& Aumento durante a colheita & $36,0 \pm 16,5$ & $34,0 \pm 11,7$ & $38,5 \pm 16,6$ & $39,0 \pm 14,7$ \\
\hline
\end{tabular}

Não houve diferença significativa entre os grupos $(\mathrm{P}>0,10)$. 
Avaliação dos métodos de contenção

Avaliando-se unicamente os métodos de contenção ( $\mathrm{E}$ = estação, $\mathrm{D}$ = decúbito lateral), os tratamentos em decúbito (AD e $\mathrm{MD}$ ) apresentaram um menor tempo para atingir a fração seminal $(\mathrm{P}<0,05)$ e uma maior duração da fração seminal $(\mathrm{P}<0,05)$ em segundos (Tabela 4). Porém, esses fatores não refletiram em um volume maior de sêmen $(\mathrm{P}>0,05)$. Os tratamentos em estação (AE e ME) apresentaram sêmen com aspecto mais cremoso do que os tratamentos em decúbito ( $\mathrm{P}<0,05$; Tabela 4$)$. No entanto, isto não refletiu na concentração espermática, o que entra em conflito com a classificação sugerida por MIES FILHO (1987), na qual quanto mais cremoso o sêmen maior sua densidade. Estes resultados demonstram a subjetividade da avaliação do aspecto quando correlacionado à concentração (REICHENBACH et al., 2008) e consequentemente à sua real importância no exame andrológico.

As concentrações plasmáticas de cortisol não mostraram diferenças quanto aos métodos de contenção ( $\mathrm{P}>0,05$; Tabela 5), demonstrando aumentos similares com média de $36,9 \pm 14,9 \mathrm{ng} / \mathrm{mL}$ e indicando um mesmo nível de estresse para os métodos de contenção avaliados. Os valores apresentados pelos grupos antes da colheita seminal (Tabela 3) estão entre os valores basais de concentração plasmática de cortisol relatados por KANNAN et al. (2000, 2002), que foram de 5 a $15 \mathrm{ng} / \mathrm{mL}$. A dosagem de cortisol circulante frequentemente é utilizada em avaliações de estresse e bem-estar animal (COOK, 1996; PRETRIE et al., 1996; KANNAN et al., 2002), por isso é empregada nas avaliações de estresse e dor causados pela eletroejaculação em touros (FALK, 2001; ETSON et al., 2004; SARTORI et al., 2005) e em bodes (ORTIZ-DE-MONTELLANO et al., 2006).

Procedimentos mais simples como massagem transretal, injeção intramuscular ou venopunção também provocam aumentos nas concentrações plasmáticas de cortisol (ALAM \& DOBSON, 1981). Por isso a avaliação do estresse animal pelo aumento de cortisol plasmático talvez não seja o melhor método, sendo necessária a realização de novos experimentos para avaliação de outros parâmetros mais sensíveis ao estado de estresse do animal, como a dosagem de progesterona, já utilizada em outros estudos (FALK et al., 2001; ETSON et al., 2004).
TABELA 4. Resultados (média \pm DP) da coleta de sêmen em carneiros utilizando-se os tratamentos em estação (AE e ME) ou os tratamentos em decúbito (AD e MD)

\begin{tabular}{|c|c|c|}
\hline & \multicolumn{2}{|c|}{ Métodos de contenção } \\
\hline & $\begin{array}{c}\text { Estação } \\
\text { AE e ME }\end{array}$ & $\begin{array}{c}\text { Decúbito } \\
\text { lateral } \\
\text { AD e MD }\end{array}$ \\
\hline Nível de estresse (0 a 3) & $1,2 \pm 0,4$ & $1,3 \pm 0,5$ \\
\hline $\begin{array}{l}\text { Tempo para atingir fração } \\
\text { seminal (s) }\end{array}$ & $71 \pm 44,1^{\mathrm{a}}$ & $53,8 \pm 53,5^{b}$ \\
\hline Tempo de fração seminal (s) & $67 \pm 26,8^{b}$ & $77,7 \pm 33,3^{a}$ \\
\hline $\begin{array}{l}\text { Tempo para atingir fração pós- } \\
\text { seminal (s) }\end{array}$ & $138 \pm 47,3$ & $131,4 \pm 60,8$ \\
\hline $\begin{array}{l}\text { Carneiros com exposição de } \\
\text { pênis durante a colheita (\%) }\end{array}$ & $88,9 \pm 31,7$ & $91,2 \pm 28,6$ \\
\hline Volume do ejaculado (mL) & $1,2 \pm 0,8$ & $1,3 \pm 0,9$ \\
\hline Aspecto* & $2,3 \pm 0,9^{\mathrm{a}}$ & $2 \pm 0,8^{b}$ \\
\hline Turbilhonamento (1- 5) & $3,5 \pm 1,3$ & $3,2 \pm 1,4$ \\
\hline Motilidade progressiva (\%) & $66,8 \pm 22,1$ & $62,4 \pm 26$ \\
\hline Vigor (1-5) & $3,5 \pm 1$ & $3,3 \pm 1,3$ \\
\hline $\begin{array}{l}\text { Total de patologias } \\
\text { espermáticas }(\%)\end{array}$ & $13,6 \pm 9,6$ & $14,3 \pm 10,9$ \\
\hline $\begin{array}{l}\text { Total de espermatozoides no } \\
\text { ejaculado (bilhões) }\end{array}$ & $\begin{array}{c}1996,4 \pm \\
2176,1\end{array}$ & $2265 \pm 2933$ \\
\hline
\end{tabular}

$\mathrm{AE}=$ automático em estação;

$\mathrm{ME}=$ manual em estação;

$\mathrm{AD}=$ automático em decúbito

$\mathrm{MD}=$ manual em decúbito

${ }^{a, b}$ Diferença entre os grupos $(\mathrm{P} \leq 0,05)$.

*1 - aquoso, 2 - leitoso, 3 - cremoso (ALK et al., 2001; ETSON et al., 2004).

TABELA 5. Concentração plasmática de cortisol (média \pm DP) em carneiros com sêmen coletado em estação (E) ou em decúbito (D)

\begin{tabular}{llcc}
\hline & & \multicolumn{2}{c}{ Métodos de contenção } \\
\hline & & Estação & $\begin{array}{c}\text { Decúbito } \\
\text { lateral }\end{array}$ \\
\hline & Antes da coleta & $11,2 \pm$ & $11,4 \pm 8,4$ \\
Cortisol & seminal & 12,1 & \\
plasmático & Após a coleta & $47,6 \pm$ & $48 \pm 13,1$ \\
(ng/mL) & seminal & 19,3 & $36,6 \pm$ \\
& Aumento durante & $37,2 \pm$ & 13,4 \\
\hline
\end{tabular}

Não houve diferença significativa entre os grupos $(\mathrm{P}>0,05)$ pelo Teste de Tukey. 
O emprego da EE nem sempre gera uma coleta bem-sucedida (DZIUK et al., 1954; MARCO-JIMÉNEZ et al., 2005). MARCO-JIMÉNEZ et al. (2005) relataram uma menor eficiência na obtenção de ejaculados com o uso da EE em relação ao uso da vagina artificial, atribuída às contaminações do ejaculado com urina ou pela falta de resposta à estimulação elétrica. Segundo DZIUK et al. (1954), a EE afetaria o reflexo de ejaculação como uma consequência da agressividade da técnica, estresse ou por estimulação insuficiente na mucosa retal, no entanto, os resultados obtidos neste estudo foram satisfatórios e demonstraram a eficácia da EE.

\section{CONCLUSÕES}

O aparelho manual apresentou desempenho similar ao automático considerando-se os parâmetros avaliados no presente estudo, assim como os métodos de contenção utilizados para a coleta. Uma vantagem observada no aparelho automático em relação ao manual foi a maior facilidade de procedimento. Com isto, a escolha do método de coleta deverá ser sempre avaliada pelo médico veterinário de acordo com a sua experiência e a disponibilidade de auxiliares.

\section{REFERÊNCIAS}

ALAM, M. G. S.; DOBSON, H. Effect of various veterinary procedures on plasma concentrations of cortisol, luteinizing hormone and prostaglandin F2-alpha metabolite in the cow. The Veterinary Record, v. 118, p. 7-10, 1986 (Abstract).

BARTH, A. D.; BOWMAN, P. A. The sequential appearance of sperm abnormalities after scrotal insulation or dexamethasone treatment in bull. The Canadian Veterinary Journal, v. 35, p. 93-102, 1994.

CARTER, P. D.; HAMILTON, P. A.; DUFTY, J. H. Eletroejaculation in goats. Australian Veterinary Journal, v. 67, p. 91-93, 1990.

CBRA. Colégio Brasileiro de Reprodução Animal. Manual para exame andrológico e avaliação de sêmen animal. 2. ed. Belo Horizonte: CBRA, 1998. 49 p. Disponível em: $<$ htt://www.sebraemg.com.br/parceiros/cbra.htm>

COOK, C. J. Basal and stress response cortisol levels and stress avoidance learning in sheep (Ovis aries). The New Zealand Veterinary Journal, v. 44, p. 162-163, 1996.

COOPER, C.; EVANS, A. C. O.; COOK, S.; RAWLINGS, N. C. Cortisol, progesterone and beta-endorphin response to stress in calves. Canadian Journal of Animal Science, v. 95, p. 197-201, 1995.

DZIUK, S. P.; GRAHAM, F. E.; DONKER, D. J.; MARION, B. G.; PETERSONEW. Some observations incollection of semen from bulls, goats boars and rams by electrical stimulation. Veterinary Medicine, v. 69, p. 455-458, 1954.

ETSON, C. J.; WALDNER, C. L.; BARTH, A. D. Evaluation of a segmented rectal probe and caudal epidural anesthesia for electroejaculation of bulls. The Canadian Veterinary Journal, v. 45, p. 235-240, 2004.

FALK, A. J.; WALDNER, C. L.; COTTER, B. S.; GUDMUNDSON, J.; BARTH, A. D. Effects of epidural lidocaine anesthesia on bulls during eletroejaculation. The Canadian Veterinary Journal, v. 42 , p. 116-120, 2001

JEPHCOTT, E. H.; MCMILLEN, I. C. A comparison of the effects of electroimobilisation and/or shearing procedures on ovine plasma concentracion of beta-endorphin/beta-lipoprotein and cortisol. Research in Veterinary Science, v. 43, p. 97-100, 1987.

JIMÉNEZ, F. M.; PUCHADES, S.; GADEA, J.; VICENTE, J. S.; VIUDES-DE-CASTRO, M. P. Effect of semen collection method on pre- and post- thaw Guirra ram spermatozoa. Theriogenology, v. 64 , p. $1756-1765,2005$.

KANNAN, G.; TERRIL, T. H.; KOUAKOU, B.; GAZAL, O.S.; GELAYE, S.; AMOAH, E. A.; SAMAKE, S. transportation of goats: effects on physiological stress responses and live weight loss. Journal of Animal Science, v. 78, p. 1450-1457, 2000.

KANNAN, G.; TERRIL, T. H.; KOUAKOU, B.; GELAYE, S.; AMOAH, E. A. Simulated preslaughter holding and isolation effects on stress responses and live weight shrinkage in meat goats. Journal of Animal Science, v. 80, p. 1771-1780, 2002.

MARDEN, W. G. R. New advances in the eletroejaculation off bull. Journal of Dairy Science, v. 37, p. 225-561, 1954.

MIES FILHO, A. Inseminação artificial. 6. ed. Porto Alegre: Editora Sulina, v. 2, 1987. 423 p.

MOSURE, W. L.; MEYER, R. A.; GUDMUNDSON, J.; BARTH, A. D. Evaluation of possible methods to reduce pain associated with eletroejaculation in bulls. The Canadian Veterinary Journal, v. 39 , p. $504-506,1998$.

ORTIZ-DE-MONTELLANO, M.; GALINDO-MALDONADO, F.; CAVAZOS-ARIZPE, E. O.; AGUAYO-ARCEO, A. M.; TORRESACOSTA, J. F. J.; ORIHUELA, A. Effect of electro-ejaculation on the serum cortisol response of Criollo goats (Capra hircus). Small Ruminant Research, v. 69, p. 228-231, 2007. 
PALMER, C. W. Walfare aspects of theriogenology: investigating alternatives to eletroejaculation of bulls. Theriogenology, v. 64, p. 469-479, 2005.

PETRIE, H. J.; MELLOR, D. J.; STAFFORD, K. J.; BRUCE, R. A.; WARD, R. N. Cortisol responses of calves to two methods of tail docking used with or without local anaesthetic. The New Zealand Veterinary Journal, v. 44, p. 4-8, 1996.

REICHENBACH, H. D.; MORAES, J. C. F.; NEVES, J. P. Tecnologia do sêmen e inseminação artificial em bovinos. In: GONÇALVES, P. B. D.; FIGUEIREDO, J. R.; FREITAS, V. J. F. Biotécnicas aplicadas à reprodução animal. 2. ed. São Paulo: Editora Rocca, 2008. p. 57-82.

ROBERTSON, I. S. Effect of different methods of castration on behavior and plasma cortisol in calves of three ages. Research in Veterinary Science, v. 56, p. 8-17, 1994.
SARTORI, R.; SILVA, T. A. S. N.; GUIMARÃES NETO, A. G.; MALAQUIAS, D. C. Coleta de sêmen em touros utilizando diferentes eletroestimuladores. In: CONGRESSO BRASILEIRO DE REPRODUÇÃO ANIMAL, 16., 2005, Goiânia. Anais... Goiânia, 2005. Disponível em: $<$ htt://www.cbra.org.br $>$

WELSH, T. H. JR.; JOHNSON, B. H. Stress induced alterations in secretion of corticosteroids, progesterone, luteinizing hormone, and testosterone in bulls. The Canadian Veterinary Journal, v. 39, p. 1-3, 1998

WELSH, T. H.; JOHNSON, B. H. Stress-induced alterations in secretion of corticosteroids, progesterone, luteinizing hormone, and testosterone in bulls. Endocrinology, v. 109, p. 185-190, 1981.

Protocolado em: 4 jun. 2008. Aceito em: 9 dez. 2009. 